\title{
Les carrières de pierre dans la vallée du Rhône
}

Jérôme Nicault

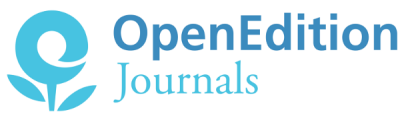

Édition électronique

URL : http://journals.openedition.org/adlfi/1715

ISSN : 2114-0502

Éditeur

Ministère de la Culture

Référence électronique

Jérôme Nicault, "Les carrières de pierre dans la vallée du Rhône », ADLFI. Archéologie de la France -

Informations [En ligne], Rhône-Alpes, mis en ligne le 01 mars 2008, consulté le 10 décembre 2020.

URL : http://journals.openedition.org/adlfi/1715

Ce document a été généré automatiquement le 10 décembre 2020.

(c) Ministère de la Culture et de la Communication, CNRS 


\title{
Les carrières de pierre dans la vallée du Rhône
}

\author{
Jérôme Nicault
}

\section{Identifiant de l'opération archéologique : 9739}

Date de l'opération : 2008 (PT)

1 Les prospections ont succédé à un long travail de dépouillement des archives départementales de l'Ardèche et de la Drôme, d'enquêtes bibliographique, toponymique et grâce à l'aide de sites internet (atlas des meulières et BRGM). Avant le travail de terrain ce n'était pas moins de 221 carrières qui étaient référencées pour 116 communes ardéchoises et 243 carrières pour 107 communes drômoises. Les carrières ainsi documentées peuvent être de pierres à bâtir, de granulats, de meules, de sable, de pierres à chaux, à gypse (plâtre), à enrochements, mais aussi, à ocre, à poterie (argile). Il est parfois précisé la façon dont s'exécute l'extraction, mais aussi le type de carrière : à ciel ouvert en fosse, en palier, en tranchée ou encore souterraine, par puits, galeries, hagues et bourrage et à piliers tournés, etc.

2 Les prospections ont permis d'étudier 11 carrières de pierre de l'Ardèche et 20 de la Drôme. II existe un réel décalage entre les deux départements qui provient du fait qu'il y a dans la Drôme deux fois plus de carrières de pierre à bâtir que dans l'Ardèche. Le travail s'est concentré sur les carrières de pierres à bâtir et à meules car cela entre dans le cadre d'une thèse concernant les carrières de pierre dans la vallée du Rhône de l'An Mil à 1900.

\section{Alixan, une carrière symbole de l'extraction médiévale}

II est déjà acquis qu'au Moyen Âge presque toutes les communautés villageoises ont leur propre carrière de pierre à bâtir, mais aussi leur meulière. L'exemple du village d'Alixan est assez éloquent puisque il y a sur la commune 3 carrières de molasse médiévales (grès argilo-calcaire à grains moyens et fins). Déjà occupé dans l'Antiquité (Amis du Vieil 
Alixan, 2007). la commune est citée en 915 dans le cartulaire de la collégiale SaintBarnard de Romans (Chevallier, 1898). De récentes fouilles archéologiques et sondages réalisés au coeur du village circulaire médiéval ont révélé la présence d'un habitat dès avant l'An Mil (Goy, Gisclon, Remy, 2001). L'extraction des pierres s'est effectuée, surtout autour du piton central (butte de molasse) à ciel ouvert, mais aussi en souterrain sous cette même butte. Cette exploitation souterraine s'est faite sous la forme de plusieurs chambres d'extractions qui s'organisent autour d'un pilier tourné, mais aussi par une longue galerie de $13 \mathrm{~m}$. De petites niches en partie noircies par la suie indique la position de lampes à huile, de bougies ou de flambeaux. Les traces d'outils sont nombreuses et permettent de déterminer à la fois le mode d'extraction, mais aussi les outils utilisés par les carriers. L'exploitation se faisait par paliers successifs de $0,3 \mathrm{~m}$ à $0,4 \mathrm{~m}$ d'épaisseur sur l'ensemble de la hauteur de la carrière $(2,2 \mathrm{~m}$ en moyenne sauf dans la galerie où la hauteur n'atteint par endroit que 1,5 m). Les coups de pics des carriers (ou peut-être d'escoudes) sont nombreux ainsi que des traces laissées par une scie, mais sans doute lors d'un essai d'extraction postérieur. Cette extraction n'a laissé aucune documentation en archive (il est seulement fait mention au $\mathrm{XV}^{\mathrm{e}} \mathrm{s}$. de balmes), mais les techniques, les traces d'outils et la faible ampleur de la carrière supposent une utilisation médiévale et ce, antérieurement au XlV $\mathrm{s}$. L'ensemble occupe une superficie de $180 \mathrm{~m}^{2}$ et a livré un cubage de pierre d'environ $368 \mathrm{~m}^{3}$. D'autres analyses de carrières m'ont indiqué que $\mathrm{d}^{\prime} 1 \mathrm{~m}^{3}$ de pierres extraites on retire $0,6 \mathrm{~m}^{3}$ de pierres à bâtir, ainsi à Alixan la carrière souterraine aurait livré $220 \mathrm{~m}^{3}$ de pierres à bâtir et $148 \mathrm{~m}^{3}$ de déchets de taille. Ce volume est très insuffisant pour les besoins du village d'où la présence d'autres carrières sur la commune, mais aussi de pierres d'importations d'origine plus ou moins lointaine.

\section{La fin des carrières locales et l'émergence des grandes carrières régionales}

4 À Alixan, on trouve dans les constructions des pierres provenant de Châteauneuf-surIsère, du Vercors et de l'Ardèche (carrière de Crussol). Les micro-carrières villageoises sont progressivement abandonnées et supplantées au cours du Moyen Âge par quelques sites d'extractions majeurs qui pour la plupart étaient connus et exploités dès l'Antiquité. On citera dans ce cadre les bassins carriers drômois, celui de Châteauneuf-sur-Isère qui couvre par moins de $350 \mathrm{~km}^{2}$, celui de Saint-Paul-Trois-Châteaux sur $280 \mathrm{~km}^{2}$ et en Ardèche celui de Chomerac $\left(90 \mathrm{~km}^{2}\right)$. Ces grands bassins carriers vont s'imposer par la qualité de la pierre extraite (facilité d'extraction et de taille, couleur, type), la proximité d'un moyen de transport (le Rhône et l'Isère) facilitant l'exportation de cette ressource, mais aussi le coût de l'extraction. À ce titre les carrières de la Montagne Sainte-Juste de Saint-Paul-Trois-Châteaux et de Saint-Restitut sont symboliques du tournant industriel que prennent certaines carrières au milieu du $\mathrm{XIX}^{\mathrm{e}} \mathrm{s}$. Des investissements sont réalisés en terme de transport moderne (le baron Dubord puis Louis Favre qui lui succède ont réalisé un chemin de fer de type Décauville qui parcourt le plateau, une rampe inclinée qui permet de descendre les pierres jusqu'à Saint-Paul et une voie de chemin de fer qui relie Saint-Paul à la gare du Paris-Lyon-Méditerranée de Pierrelatte), mais aussi en modernisant les moyens d'extractions (scie crocodile, extraction en galerie, utilisation de cric pour déplacer les blocs). Les pierres de la Montagne Sainte-Juste sont alors abordables, de très bonne qualité et exportées non seulement dans tout le département 
de la Drôme, mais aussi à Avignon, Lyon, Marseille, Grenoble, Saint-Etienne et même New-York.

5 L'évolution constatée pour les carrières de pierre à bâtir a été faite par Alain Belmont (Belmont, 2006). Il avait montré que les meulières étaient présentes dans presque tous les villages au début du Moyen Âge et qu'elles avaient disparu au cours des siècles, remplacées par des meules provenant de carrières à vocation régionale et parfois plus lointaine comme celle de la Ferté-sous-Jouarre.

6 À l'avenir on pourrait entreprendre quelques sondages dans des carrières et essayer ainsi de mieux comprendre, tout à la fois leur organisation spatiale, mais aussi les techniques employées pour l'extraction. Des analyses plus fines des fronts de taille et des traces laissées par les outils permétraient peut-être de mieux définir une chronologie ou d'établir tout du moins une chronologie relative propre aux carrières rhodaniennes. Enfin, des analyses physico-chimiques seraient utiles afin de mieux comprendre comment les anciens opéraient leur choix entre la multitude des pierres et les carrières disponibles.

7 À moyen terme de nouvelles prospections archéologiques thématiques seront étendues dans les autres départements rhodaniens.

8 Nicault Jérôme

\section{INDEX}

Index chronologique : Antiquité, ép. contemporaine, Moyen Âge

Index géographique : Rhône-Alpes, Drôme (26), Alixan

Thèmes : ocre

operation Prospection thématique (PRT)

\section{AUTEUR}

\section{JÉRÔME NICAULT}

Chercheur 